\title{
Tres nuevos registros del genero Carex (Cyperaceae) para el Perú y adiciones a la flora andina del departamento de Huánuco
}

\section{Three new records of the genus Carex (Cyperaceae) from Peru and additions to the Andean flora of the Huánuco department}

\section{Flor de María Salvador, María Ángeles Alonso y Segundo Ríos}

Instituto Universitario de Investigación- CIBIO, Universidad de Alicante, Apdo. de Correos 99 E-03080, Alicante, España. E-mail E-03080, Alcante, Espana. E-mail yahoo.es

Presentado: 05/02/2008 Aceptado: 29/12/2008 Publicado online: 26/02/2009

\begin{abstract}
Three new records from Peru (Carex humahuacaensis G.A. Wheeler, Carex peucophila T. Holm y Carex pygmaea Boeck.), and 75 new records of vascular plants are reported for Huánuco department. Its habitat, distribution and the conservation status of endemisms such as: Caiophora cirsiifolia C. Presl, Carex hebetata Boott and Senecio macrorrhizus Wedd. are commented.
\end{abstract}

Keywords: Andes, flora, chorology, endemisms, Peru.

\section{Resumen}

Tres nuevos registros para el Perú (Carex humahuacaensis G.A. Wheeler, Carex peucophila T. Holm y Carex pygmaea Boeck.) y 75 nuevos registros para la flora del departamento de Huánuco son dados a conocer. Se informan datos sobre el hábitat, distribución y en el caso de endemismos como: Caiophora cirsiifolia C. Presl, Carex hebetata Boott, y Senecio macrorrhizus Wedd. su estado actual de conservación.

Palabras clave: Andes, flora, corología, endemismos, Perú.

\section{Presentación}

El presente trabajo es la segunda y última contribución a la flora del Perú y del departamento de Huánuco iniciada con los resultados publicados por Salvador et al. en el año 2006. El área de estudio se circunscribe en la subcuenca del río Lauricocha situada en la vertiente atlántica de los Andes centrales, a una elevación entre 3845 m y 4795 m y cuyos límites están dados por las coordenadas geográficas: $10^{\circ} 31^{\prime}-10^{\circ} 51^{\prime} \mathrm{S}$ y $76^{\circ} 65^{\prime}$ $76^{\circ} 79^{\prime} \mathrm{W}$. Esta área ha sido descrita ampliamente en Salvador et al. (2006).

Las expediciones de campo fueron realizadas en el periodo 2002-2006, los cuales coincidieron con la estación seca y más fría del año (agosto-septiembre) y con el final de la estación lluviosa (mayo). Para este estudio, el material y la base de datos de las especies dudosas y conflictivas, principalmente representada por la familia Cyperaceae, fueron consultados con especialistas y en los siguientes herbarios: Field Museum of Natural History (F), Missouri Botanical Garden (MO), University of Michigan $(\mathrm{MICH})$ y New York Botanical Garden (NY).

La nomenclatura y taxonomía siguieron los criterios utilizados en el Catálogo de las plantas con flores y Gimnospermas del Perú (Brako \& Zarucchi, 1993), actualizados en la base de datos del herbario MO (Missouri Botanical Garden, 2007). Por otro lado, en la determinación de los taxones se ha recurrido principalmente a la siguientes literatura: Macbride (1936 - 1967), Wheeler 1989, Reznicek 1993, Koyama \& Guaglianone, 1987, Seberg 1994, Dhooge \& Goetghebeur (2002 y 2004), Al-Shehbaz (1990 y 2004), Dhooge, Goetghebeur \& Muasya, (2003).

Los pliegos de las colectas realizadas fueron depositados en los herbarios de la Universidad de Alicante $(\mathrm{ABH})$, University of Michigan (MICH) y de la Universidad Nacional Mayor de San Marcos (USM).

Los resultados han sido dispuestos a manera de un catálogo comentado donde se informa sobre los pliegos recolectados, su hábitat y distribución. La distribución fitogeográfica sigue a Rivas-Martínez et al. (1999) mientras que la distribución en el
Perú esta de acuerdo con la división política por departamentos. Como novedad, se ha considerado oportuno recoger en el catálogo florístico el estado de conservación de los endemismos registrados según el libro rojo de las especies endémicas del Perú (León et al. 2006), el cual se enmarca dentro de los criterios de la UICN (2001).

\section{Resultados y discusión}

El estudio realizado, fundamentalmente en los humedales de la subcuenca del río Lauricocha y en algunas zonas colindantes (prados, pajonales, laderas rocosas y zonas riparias), aporta a la flora peruana, en esta segunda contribución, con un total de 75 nuevos registros para el departamento de Huánuco y de los cuales tres son nuevos registros para Perú. Los nuevos registros para Perú corresponden a especies del género Carex (C. humahuacaensis, C. pygmaea, C. peucophila) de la familia Cyperaceae, todas ellas localizadas en turberas. Probablemente, muchas de las especies citadas de esta familia han pasado por desapercibidas en estos hábitats. Por ejemplo, algunas especies de la tribu Scirpeae, pertenecientes a los géneros Oreobolus, Oreobolopsis, Phylloscirpus y Zameioscirpus y especies altoandinas de Carex, sección Abditispicae (i.e. C. humahuacaensis), debido a su pequeño porte, son pasadas por alto (Wheeler, 2002) y además son típicamente confundidas entre ellas mismas y muchas veces con Distichia muscoides. Sin duda es de suponer que aún falta mucho por descubrir en los pliegos de herbarios y en las zonas de turberas altoandinas del Perú.

La zona estudiada alberga a su vez cinco endemismos, cuatro de ellos localizados en los Andes peruanos (Tarasa cerratei, Carex hebetata, Caiophora cirsiifolia y Senecio macrorrhizus) y un endemismo registrado en los Andes de Huánuco (Pilea dombeyana). Cuatro de los endemismos citados: Tarasa cerratei, Carex hebetata, Pilea dombeyana y Senecio macrorrhizus se localizan en los humedales de la subcuenca de Lauricocha. De ahí resalta la importancia de señalar a los humedales estudiados, y en general a los humedales altoandinos, como hábitats relevantes igualmente para la conservación de especies endémicas de plantas peruanas. 
Por último, es necesario destacar que para la subcuenca del río Lauricocha las amenazas referidas a los estados de conservación se ciernen en dos aspectos, uno relacionado con la mala gestión de la ganadería y el otro con el desarrollo de la minería polimetálica (Salvador 2008). Esta situación representa sólo una pequeña muestra de lo que sucede actualmente en las cuencas altoandinas del Perú (Salvador \& Cano, 2002) y lo grave es que no se han encontrado estudios publicados, de este tipo, en zonas con actividad minera. En consecuencia, éstas áreas necesitan ser estudiadas para valorar y aumentar el conocimiento de la biodiversidad, con el fin de incentivar otros estudios ambientales y lograr una buena gestión sostenible para mejorar la calidad de vida de los pobladores y el estado de conservación de los hábitats.

\section{Catálogo comentado}

\section{PTERIDOPHYTA}

\section{THELYPTERIDACEAE}

\section{Thelypteris demissa A. R. Sm.}

PERÚ, Huánuco: bajada de riachuelo rumbo a laguna Tactapata primera, $4000 \mathrm{~m}$, 12.VIII.2003, F. Salvador; $M$. A. Alonso \& J. Monerris 643 (USM); río Antacallanca, entre laguna Tinquicocha y Chuspi, $4250 \mathrm{~m}, 12$.V.2004, F. Salvador; S. Rios \& E. Arias 896 (USM).

Planta fisurícola, destaca su presencia en paredes rocosas y rezumantes de riberas.

Distribución.- comprende los territorios de la puna de Bolivia y Perú. Hasta el momento registrada en el departamento de Amazonas, con esta colección se amplia su extensión a los Andes centrales.

\section{MagnOLIOPHYTA}

\section{Amaranthaceae}

\section{Alternanthera lupulina Kunth}

PERÚ, Huánuco: laguna Lauricocha, lado norte, $3845 \mathrm{~m}$, 9.VIII.2002, F. Salvador; M. A. Alonso \& M. Rodríguez 479 (USM).

Se desarrolla en riberas, laderas arenoso-rocosas y bajo matorrales.

Distribución.- especie propia de la provincia de la Puna Peruana, registrada en los Andes peruanos. Otras localidades citadas: Ancash, Cajamarca, Junín, Lima, La Libertad, Pasco, Puno.

\section{Asteraceae}

3. Ageratina azangaroensis (Sch. Bip. ex Wedd.) R.M. King \& H. Rob.

PERÚ, Huánuco: laguna Lauricocha, lado suroeste, 3845 m, 7.VIII.2002, F. Salvador; M. A. Alonso \& M. Rodríguez 388 (USM); río Antacallanca, entre laguna Tinquicocha y Chuspi, 4250 m, 12.V.2004, F. Salvador; S. Rios \& E. Arias 893 (USM).

Habita riberas y matorrales.

Distribución.- localizada en los Andes de Bolivia, Ecuador y Perú. Con buena representación en el territorio peruano, ha sido colectada en los siguientes departamentos: Ancash,
Arequipa, Ayacucho, Cajamarca, Cusco, Lambayeque, Lima, La Libertad, Pasco y Puno.

\section{Baccharis genistelloides (Lam.) Pers.}

PERÚ, Huánuco: laguna Lauricocha, lado suroeste, $3845 \mathrm{~m}$, 7.VIII.2002, F. Salvador; M. A. Alonso \& M. Rodríguez 396 (USM); bajada de riachuelo rumbo a laguna Tactapata 1, 4000 m, 12.VIII.2003, F. Salvador; M. A. Alonso \& J. Monerris 641 (USM), laguna Pucacocha, nacimiento de río Antacallanca, 4350 m, 11.V.2004, F. Salvador; S. Ríos \& E. Arias 837 (USM).

Se encuentra en riberas, matorrales y laderas rocosas.

Distribución.- Neotropical-Austroamericana. Ampliamente distribuida en el territorio peruano.

\section{Baccharis tricuneata (L. f.) Pers.}

PERÚ, Huánuco: ladera en río que va hacia Añaspampa, 4000 m, 14.VIII.2003, F. Salvador; M. A. Alonso \& J. Monerris 694 (USM); río Antacallanca, entre laguna Tinquicocha y Chuspi, 4250 m, 12.V.2004, F. Salvador; S. Rios \& E. Arias 891 (USM).

Suele habitar zonas de riberas y matorrales.

Distribución.- Neotropical-Austroamericana. En el Perú registrada en los departamentos de Ancash, Apurímac, Cajamarca, Cusco, Junín, Lima, La Libertad, Puno y Tacna.

\section{Dendrophorbium usgorense (Cuatrec.) C. Jeffrey}

PERÚ, Huánuco: río Lauricocha, 3326 m, 8.VIII.2003, F. Salvador; M. A. Alonso \& J. Monerris 554 (USM).

Aparece en laderas pedregosas y húmedas de riberas.

Distribución.- se restringe hasta el momento a los Andes de Ecuador y Perú. Con esta colección se amplia su distribución hacia la zona central. Se ha registrado en los departamentos de Amazonas, Ancash, Cajamarca y La Libertad.

\section{Gnaphalium polium Wedd.}

PERÚ, Huánuco: laguna Lauricocha, 3865 m, 11.VIII.2002, F. Salvador; M. A. Alonso \& M. Rodríguez 536 (USM).

Normalmente se ha observado en laderas arenoso-pedregosas y bajo matorrales.

Distribución.- circunscrita a las zonas altoandinas de Bolivia y Perú. En Perú ha sido colectada en los siguientes departamentos: Ancash, Cajamarca, Cusco, Junín, Lima, La Libertad y Puno.

8. Lasiocephalus campanulatus (Sch. Bip. ex Klatt) Cuatrec.

PERÚ, Huánuco: río Lauricocha, 3326 m, 8.VIII.2003, F. Salvador; M. A. Alonso \& J. Monerris 557 (USM).

Habita en zonas de riberas junto con Polylepis aff. incana.

Distribución.- puna de Bolivia y Perú. Escasamente recolectada, registrada en los departamentos de Ancash, Cusco y La Libertad.

9. Paranephelius ovatus A. Gray ex Wedd.

PERÚ, Huánuco: laguna Chuspi, cerca de orilla, $4100 \mathrm{~m}$, 12.VIII.2003, F. Salvador; M. A. Alonso \& J. Monerris 613 (USM). 
Escasamente observada, suele colonizar derrubios.

Distribución.- hasta el momento registrada en las zonas altoandinas de Bolivia y Perú. Con esta colección se amplia su distribución en los Andes centrales peruanos. Citada en los departamentos de Ancash, Cajamarca, Cusco y Junín.

10. Perezia multiflora (Bonpl.) Less.

PERÚ, Huánuco: campamento Raura, laguna Caballococha, orilla suroeste, 4575 m, 8.V.2004, F. Salvador; S. Ríos \& E. Arias 738 (ABH, USM).

Suele crecer sobre suelos arenosos, ligeramente húmedos y nitrificados.

Distribución.- localizada en los territorios andinos de Argentina, Bolivia, Chile, Ecuador y Perú. Ampliamente distribuida en los Andes peruanos.

\section{Senecio candollei Wedd.}

PERÚ, Huánuco: laguna Nińococha bajo, 4795 m, 7.V.2004, F. Salvador; S. Ríos \& E. Arias 733 (USM); a $100 \mathrm{~m}$ de puesto de control Santa Rosa (campamento Raura), ladera abajo, 4650-4700 m, 13.V.2004, F. Salvador; S. Ríos \& E. Arias 927 (USM).

Habita en turberas y laderas con suelos crioturbados situados por encima de los $4600 \mathrm{~m}$.

Distribución.- hasta la actualidad exclusiva de la puna de Bolivia y Perú. Escasamente colectada en el Perú, registrada en los departamentos de Lima y Puno.

\section{Senecio comosus Sch. Bip.}

PERÚ, Huánuco: a $100 \mathrm{~m}$ de puesto de control Santa Rosa (campamento Raura), 4700-4750 m, 13.V.2004, F. Salvador; S. Ríos \& E. Arias 935 (ABH, USM).

Crece sobre laderas pedregosas situadas por encima de $4500 \mathrm{~m}$.

Distribución.- distribuida en los Andes de Bolivia, Ecuador y Perú. En el territorio peruano se localiza en Ancash, Apurímac, Cusco, Junín, La Libertad y Puno.

\section{Senecio culcitioides Sch. Bip.}

PERÚ, Huánuco: bajada de riachuelo rumbo a laguna Tactapata primera, 4000 m, 12.VIII.2003, F. Salvador; M. A. Alonso \& J. Monerris 642 (ABH, USM); laguna Pucacocha, nacimiento de río Antacallanca, 4350 m, 11.V.2004, F. Salvador; S. Ríos \& E. Arias 838 (USM), laguna Tinquicocha, parte central, orilla oeste, bajo, 4350 m, 12.V.2004, F. Salvador; S. Rios \& E. Arias 876 (USM).

Habita en riberas y se encuentra junto con gramíneas asociadas al agua.

Distribución.- hasta el momento restringida a los territorios de Ecuador y Perú. Pobremente colectada en el Perú, registrada para los departamentos de Ancash, Lima y La Libertad.

\section{Senecio evacoides Sch. Bip.}

PERÚ, Huánuco: campamento Raura, laguna Niñococha alto, 4795 m, 7.V.2004, F. Salvador; S. Ríos \& E. Arias 731 (USM); laguna Pucacocha, nacimiento de río Antacallanca, $4350 \mathrm{~m}$, 11.V.2004, F. Salvador; S. Ríos \& E. Arias 839 (USM).
Suele encontrarse en riberas y laderas de derrubios, localizadas por encima de $4300 \mathrm{~m}$.

Distribución.- hasta la actualidad registrada en los territorios andinos de Argentina, Bolivia y Perú. En el Perú ha sido colectada en los siguientes departamentos: Ancash, Arequipa, Ayacucho, Cusco, Junín, Lima, Moquegua, Pasco y Puno.

\section{Senecio graveolens Wedd.}

PERÚ, Huánuco: campamento Raura, laguna Niñococha alto, 4795 m, 7.V.2004, F. Salvador; S. Rios \& E. Arias 730 (USM).

Se desarrolla en laderas con derrubios situados sobre $4600 \mathrm{~m}$.

Distribución.- distribuida en los Andes de Argentina, Bolivia, Perú y Chile. Escasamente colectada en el Perú.

\section{Senecio macrorrhizus Wedd.}

PERÚ, Huánuco: laguna Lauricocha, 3845 m, 5.VIII.2002, F. Salvador; M. A. Alonso \& M. Rodríguez 347 (USM); Laguna Patarcocha, 4100 m, 11.VIII.2003, F. Salvador; M. A. Alonso \&J. Monerris 606 (USM); ladera arenosa, Añaspampa a 4 $\mathrm{km}$ de Antacallanca, en carretera hacia Lauricocha, $4000 \mathrm{~m}$, 14.VIII.2003, F. Salvador; M. A. Alonso \& J. Monerris 700 (USM); laguna Tinquicocha, orilla oeste, $4350 \mathrm{~m}, 12 . \mathrm{V} .2004$, F. Salvador; S. Ríos \& E. Arias 875 (USM).

Se encuentra en turberas, prados y pajonales asociados al agua.

Distribución.- endemismo de la provincia de la Puna Peruana, solamente registrada en Perú. Distribuida en los Andes centrales de Perú, citada para los departamentos de Ancash, Cajamarca, Junín y Pasco.

Estatus de conservación.- DD. Esta especie pertenece a un complejo que requiere estudios taxonómicos y cuyo estatus no es claro (Beltrán et al., 2006).

\section{Senecio rbizomatus Rugby}

PERÚ, Huánuco: campamento Raura, laguna Niñococha bajo, 4795 m, 7.V.2004, F. Salvador; S. Ríos \& E. Arias 732 (USM).

Habita laderas con derrubios situados por encima de $4600 \mathrm{~m}$.

Distribución.- hasta el momento exclusiva de la puna de Bolivia y Perú. En el Perú escasamente colectada, citada para los departamentos de Ancash, Cusco y La Libertad.

\section{Senecio serratifolius (Meyen \& Walp.) Cuatrec.}

PERÚ, Huánuco: a $100 \mathrm{~m}$ de puesto de control Santa Rosa (campamento Raura), ladera abajo, 4650-4700 m, 13.V.2004, F. Salvador; S. Ríos \& E. Arias 948 (USM).

Se encuentra en turberas dominadas por Distichia muscoides Nees \& Meyen y Plantago rigida Kunth sobre 4600 m.

Distribución.- distribuida en los Andes de Argentina, Bolivia, Chile y Perú. En el Perú hasta el momento citada sólo para los departamentos de Ancash, Cusco y Lima.

19. Werneria pygmaea Gillies ex Hook. \& Arn.

PERÚ, Huánuco: laguna Lauricocha, ladera este, 3845 m, 6.VIII.2002, F. Salvador; M. A. Alonso \& M. Rodríguez 376 (USM); Laguna Patarcocha, 4100 m, 11.VIII.2003, 
F. Salvador; M. A. Alonso \& J. Monerris 588 (USM); laguna Chuspi, 4150 m, 12.VIII.2003, F. Salvador; M. A. Alonso \& J. Monerris 628 (USM); a 100 m de puesto de control Santa Rosa (campamento Raura), ladera arriba, 4700-4750 m, 13.V.2004, F. Salvador; S. Ríos \& E. Arias 922 (USM); a $1 \mathrm{~km}$ de laguna Aguascocha, río Quichas, Oyón, Lima, 4016 m, 14.V.2004, F. Salvador; S. Ríos \& E. Arias 991 (USM).

Se encuentra frecuentemente en turberas, aunque también se desarrolla en prados turbosos.

Distribución.- distribuida en todos los Andes. En el Perú registrada en los departamentos de Ancash, Ayacucho, Cusco, Junín, Lima, Puno y Tacna.

20. Xenophyllum dactylophyllum (Sch. Bip.) V.A. Funk PERÚ, Huánuco: Campamento Raura, ladera, camino a laguna Niñococha bajo, 4700 m, 7.V.2004, F. Salvador; S. Rios \& E. Arias 735 (ABH, USM); a $100 \mathrm{~m}$ de puesto de control Santa Rosa (campamento Raura), 4700-4750 m, 13.V.2004, F. Salvador; S. Ríos \& E. Arias 944 (USM, ABH).

Especie propia de suelos crioturbados situados por encima de $4600 \mathrm{~m}$.

Distribución.- hasta la actualidad restringida a los territorios andinos de Bolivia y Perú. En el Perú distribuida en los Andes centrales, citada para los departamentos de Ancash, Junín, Lima y Puno.

\section{BRASSICACEAE}

\section{Descurainia athroocarpa (A. Gray) O.E. Schulz}

PERÚ, Huánuco: río Antacallanca, entre laguna Tinquicocha y Chuspi, 4250 m, 12.V.2004, F. Salvador; S. Rios \& E. Arias 900 (ABH. USM).

Se desarrolla en márgenes de lagunas sin orilla y bajo pendientes rocosas.

Distribución.- hasta el momento restringida a los Andes de Bolivia y Perú. En el Perú registrada en los departamentos de Ancash, Cusco, Junín, Lima, Pasco y Tacna.

\section{Descurainia depressa (Phil.) Prantl}

PERÚ, Huánuco: campamento Raura, laguna Santa Ana, 4681 m, 7.V.2004, F. Salvador; S. Ríos \& E. Arias 721 (ABH, USM); a $100 \mathrm{~m}$ de puesto de control Santa Rosa (campamento Raura), 4700-4750 m, 13.V.2004, F. Salvador; S. Ríos \& E. Arias 936 (ABH, USM).

Crece en márgenes de lagunas abruptas, laderas arenosopedregosas y bajo rocas situadas sobre $4600 \mathrm{~m}$.

Distribución.- hasta la actualidad exclusiva de Argentina y Perú. Escasamente colectada en el Perú, citada para los departamentos de Ancash (Recuay, cf. Monsalve \& Cano, 2005) y Lima.

\section{Descurainia leptoclada Muschl.}

PERÚ, Huánuco: río Lauricocha, 3326 m, 8.VIII.2003, F. Salvador; M. A. Alonso \& J. Monerris $564^{b}(\mathrm{ABH})$.

Habita en riberas con laderas de suelos ligeramente húmedos, acompañan matorrales de Ageratina azangaroensis (Sch. Bip. ex Wedd.) R.M. King \& H. Rob.
Pobremente recolectada en el Perú, citada para los departamentos de Ancash (Huaylas, cf. Monsalve \& Cano, 2005) y Lima.

Distribución.- se distribuye en los territorios andinos de Argentina, Bolivia, Ecuador, Perú.

\section{Weberbauera spathulifolia (A. Gray) O.E. Schulz}

PERÚ, Huánuco: a $100 \mathrm{~m}$ de puesto de control Santa Rosa (campamento Raura), 4700-4750 m, 13.V.2004, F. Salvador; S. Rios \& E. Arias 937 (ABH, USM).

Se desarrolla en laderas arenoso-pedregosa y bajo rocas situadas por encima de $4600 \mathrm{~m}$.

Distribución.- hasta el momento restringida a los Andes de Bolivia y Perú. Pobremente colectada en Perú, citada solamente para Ancash.

\section{Cyperaceae}

25. Carex boliviensis Van Heurck \& Müll. Arg.

PERÚ, Huánuco: oconal de Añaspampa, 3915 m, 12.V.2004, F. Salvador; S. Ríos \& E. Arias 910 (USM)

Habita riachuelos de poca corriente.

Distribución.- localizada en los Andes de Bolivia y Perú. En el territorio peruano citada para los departamentos de Ancash, Cajamarca, Cusco y La Libertad.

\section{Carex brachycalama Griseb.}

PERÚ, Huánuco: depresiones pantanosas entre laguna Tinquicocha y Chuspi, 4000 m, 15.VIII.2003, F. Salvador; $M$. A. Alonso \& J. Monerris 681 (USM).

Se ha observado entre pajonales asociados al agua.

Distribución.- distribuida en los Andes de Argentina, Bolivia, Ecuador, Perú y Venezuela.

\section{Carex hebetata Boott}

PERÚ, Huánuco: laguna Chuspi, 4150 m, 12.VIII.2003, F. Salvador; M. A. Alonso \& J. Monerris $614^{b}$ (USM); ibidem, 12.VIII.2003, F. Salvador; M. A. Alonso \& J. Monerris 615 (USM); subida a laguna Tactapata, $4050 \mathrm{~m}, 12$. VIII.2003, F. Salvador; M. A. Alonso \& J. Monerris 634 ; laguna Chuspi, orilla oeste, 4150 m, 9.V.2004, F. Salvador; S. Ríos \& E. Arias $759^{b}$ (USM).

Propia de comunidad de gramíneas altas asociadas al agua.

Distribución.- endemismo de registrado sólo en los Andes peruanos. Citada para los departamentos de Ancash, Junín, Lima, La Libertad y San Martín.

Estatus de conservación.- VU, B1a.

\section{Carex humabuacaensis G.A. Wheeler}

m.e.: Laguna Patarcocha, 4100 m, 11.VIII.2003 (USM), F. Salvador; M. A. Alonso \& J. Monerris 585, valle de Ańaspampa, a $5 \mathrm{~km}$ de Antacallanca, $4000 \mathrm{~m}, 14$.VIII.2003, F. Salvador; M. A. Alonso \& J. Monerris 709 (USM); laguna Lauricocha, 3845 m, 28.IX.2006, F. Salvador; J. Monerris \& R. Soto 1008 (ABH).

Suele crecer en turberas dominadas por Plantago tubulosa Decne. 
Distribución.-era conocida sólo en los Andes de Argentina (Wheeler, 1989). Con esta colección se registra por primera vez en el Perú.

\section{Carex peucophila T. Holm}

PERÚ, Huánuco: cerro Chogopata, a $5 \mathrm{~km}$ de comunidad campesina Lauricocha, 4000 m, 10.V.2004, F. Salvador; S. Ríos \& E. Arias 793 (USM).

Crece en prados y cerca de charcas temporales.

Distribución.- Mesoamérica (montaña) y región Andina. Distribuida en los Andes de Colombia y Bolivia. Con esta colección se registra por primera vez en el Perú.

\section{Carex pygmaea Boeck.}

PERÚ, Huánuco: cerro Chogopata, a $5 \mathrm{~km}$ de comunidad campesina Lauricocha, 4000 m, 10.V.2004, F. Salvador; S. Ríos \&. E. Arias 795 (USM).

Suele encontrarse en prados que sufren agostamiento.

Distribución.- hasta el momento distribuida en los Andes de Colombia y Ecuador. Con esta colección se cita por primera vez para Perú.

\section{Cyperus laevigatus L.}

PERÚ, Huánuco: Jesús, río Lauricocha, 3326 m, 9.VIII.2003, F. Salvador; M. A. Alonso \& J. Monerris 578 (USM).

Crece en suelos húmedos cercanos a riberas.

Distribución.- cosmopolita. En Perú citada para los siguientes departamentos: Arequipa, Cajamarca, Ica, Lima, La Libertad, Piura y Tacna.

32. Eleocharis albibracteata Nees \& Meyen ex Kunth PERÚ, Huánuco: laguna Tactapata primera, $4275 \mathrm{~m}$, 12.VIII.2003, F. Salvador; M. A. Alonso \& J. Monerris 651 (USM); laguna Tinquicocha, $4350 \mathrm{~m}, 13$. VIII.2003, F. Salvador; M. A. Alonso \& J. Monerris 666(USM); Laguna Patarcocha, orilla oeste, 4100 m, 9.V.2004, F. Salvador; S. Rios \& E. Arias 774 (USM); laguna Tinquicocha, orilla noroeste, a $2 \mathrm{~km}$ de laguna Chuspi, 4350 m, 9.V.2004, F. Salvador; S. Ríos \& E. Arias 782 (USM), riachuelo entre laguna Tinquicocha y Chuspi, $4200 \mathrm{~m}$, 12.V.2004, F. Salvador; S. Ríos \& E. Arias 866 (USM).

Se ubica normalmente en turberas aunque también ha sido observado en zonas pantanosas.

Distribución.- citada para los Andes de Argentina, Bolivia, Chile, Ecuador y Perú. En Perú, registrada en los departamentos de Ancash, Apurímac, Arequipa, Cusco y Puno.

\section{Oreobolopsis tepalifera T. Koyama \& Guagl.}

PERÚ, Huánuco: campamento Raura, laguna Caballococha, orilla suroeste, 4575 m, 8.V.2004, F. Salvador; S. Ríos \& E. Arias 749 (USM).

Observada ocasionalmente, suele crecer en turberas.

Distribución.- hasta el momento registrada en los Andes de Bolivia, Ecuador y Perú. En el Perú escasamente recolectada, citada sólo para Ancash.

\section{Oreobolus ecuadorensis T. Koyama}

PERÚ, Huánuco: laguna Patarcocha, 4100 m, 11.VIII.2003, F. Salvador; M. A. Alonso \& J. Monerris 600 (USM); laguna Tactapata primera, 4300 m, 12.VIII.2003, F. Salvador; M. A. Alonso \& J. Monerris 652 (USM).

Especie en almohadillado propia de turberas de Plantago tubulosa.

Distribución.- hasta la actualidad citada para los Andes de Ecuador y Perú. Escasamente recolectada en el Perú, con esta colección se amplia su distribución en la zona central, citada para los departamentos de Ancash, Pasco y San Martín.

35. Phylloscirpus acaulis (Phil.) Dhooge \& Goetgh. subsp. pachycaulis Dhooge \& Goetgh.

PERÚ, Huánuco: laguna Patarcocha, 4100 m, 11.VIII.2003, F. Salvador; M. A. Alonso \& J. Monerris 592 (USM); laguna Patarcocha, orilla oeste, 4100 m, 12.V.2004, F. Salvador; S. Ríos \& E. Arias 884 (USM).

Escasamente observada, suele desarrollarse en turberas de Distichia muscoides.

Distribución.- exclusiva de los Andes de Ecuador y Perú. Hasta el momento registrada sólo en el departamento de Puno, con esta colección se amplia su distribución hacia los Andes centrales peruanos.

36. Phylloscirpus boliviensis (Barros) Dhooge \& Goetgh.

PERÚ, Huánuco: laguna Pucacocha, nacimiento de río Antacallanca, 4350 m, 11.V.2004, F. Salvador; S. Ríos \& E. Arias 855 (USM).

Raramente colectada, se desarrolla en suelos turbosos asociados a desagües de lagunas que sufren fuerte estiaje,

Distribución.- registrada en los Andes de Bolivia, Colombia, Ecuador y Perú. En Perú escasamente recolectada citada solamente para el departamento de Ancash.

37. Phylloscirpus deserticola (Phil.) Dhooge \& Goetgh.

PERÚ, Huánuco: laguna Pucacocha, nacimiento de río Antacallanca, 4350 m, 11.V.2004, F. Salvador; S. Ríos \& E. Arias 833 (USM); laguna Pucacocha, nacimiento de río Antacallanca, 4350 m, 11.V.2004, F. Salvador; S. Ríos \& E. Arias 854 (USM); riachuelo entre laguna Tinquicocha y Chuspi, 4200 m, 12.V.2004, F. Salvador; S. Ríos \& E. Arias 862 (USM); a $50 \mathrm{~m}$ de laguna Caballococha, orilla norte, 4580 m, 13.V.2004, F. Salvador; S. Ríos \& E. Arias 969 (USM).

Suele habitar turberas dominadas por Distichia muscoides, márgenes de riachuelos y lagunas.

Distribución.- localizada en los Andes de Argentina, Bolivia, Chile, Ecuador y Perú. En Perú escasamente colectada, citada para los departamentos de Ancash y Cusco.

\section{Uncinia macrolepis Decne.}

PERÚ, Huánuco: a $100 \mathrm{~m}$ de puesto de control Santa Rosa (campamento Raura), ladera arriba, comunidad turfófila en desecación, 4700-4750 m, 13.V.2004, F. Salvador; S. Ríos \& E. Arias 929 (USM).

Especie escasamente recolectada, suele crecer en turberas dominadas por Distichia muscoides y Plantago rigida situadas sobre $4600 \mathrm{~m}$. 
Distribución.- Andino-Austroamericana. Registrada en los Andes de Argentina, Ecuador, Bolivia, Chile y Perú. En Perú registrada solamente en Junín, con esta colección se amplia su distribución hacia la zona central.

\section{Zameioscirpus muticus Dhooge \& Goetgh.}

PERÚ, Huánuco: oconal de Añaspampa, 3915 m, 10.VIII.2002, F. Salvador; M. A. Alonso \& M. Rodríguez 468 (USM); ibidem, Salvador; M. A. Alonso \& M. Rodríguez 515; ibidem 14.VIII.2003, F. Salvador, M. A. Alonso \&J. Monerris 708 (USM); laguna Patarcocha, orilla oeste, $4100 \mathrm{~m}, 12$.V.2004, F. Salvador; S. Ríos \& E. Arias 881 (USM); a $100 \mathrm{~m}$ de puesto de control Santa Rosa (campamento Raura), ladera arriba, 47004750 m, 13.V.2004, F. Salvador; S. Ríos \& E. Arias 923 (USM); a $70 \mathrm{~m}$ de puesto de control Santa Rosa (campamento Raura) cuenca Atlántica, 4650-4700 m, 13.V.2004, F. Salvador; S. Ríos \&. E. Arias 941 (USM); a $100 \mathrm{~m}$ de puesto de control Santa Rosa (campamento Raura), ladera abajo, 4650-4700 m, 13.V.2004, F. Salvador; S. Rios \& E. Arias 958 (USM); a $50 \mathrm{~m}$ de laguna Caballococha, orilla norte, oconal, $4580 \mathrm{~m}, 13$. V.2004, F. Salvador; S. Ríos \& E. Arias 971 (USM).

Especie ampliamente recolectada en turberas dominadas por Distichia muscoides y Plantago tubulosa.

Distribución.- distribuida en los Andes de Argentina, Bolivia, Chile y Perú. En Perú escasamente colectada citada para Ancash y Lima. Con esta colección se amplia su distribución en los Andes centrales peruanos.

\section{FABACEAE}

\section{Lupinus microphyllus Desr.}

PERÚ, Huánuco: laguna Patarcocha, orilla oeste, $4100 \mathrm{~m}$, 9.V.2004, F. Salvador; S. Ríos \& E. Arias 770 (USM).

Suele encontrarse en suelos turbosos asociados a bordes de lagunas.

Distribución.- citada para los Andes de Bolivia, Ecuador y Perú. En el Perú registrada en los departamentos de Ancash, Cusco, Junín y Lima.

\section{Geraniaceae}

\section{Geranium sessiliflorum Cav.}

PERÚ, Huánuco: campamento Raura, laguna Santa Ana, 4681 m, 7.V.2004, F. Salvador; S. Ríos \& E. Arias 723 (USM).

Crece en márgenes de lagunas y también bajo matorrales.

Distribución.- Andino-Austroamericana-Nuevazelandesa. En los Andes se registra en los territorios de Argentina, Bolivia, Chile, Ecuador y Perú. En el Perú se encuentra ampliamente distribuida.

\section{GrossulariaceAe}

\section{Ribes cuneifolium Ruiz \& Pav.}

PERÚ, Huánuco: laguna Lauricocha, orilla suroeste, $3845 \mathrm{~m}$, 7.VIII.2002, F. Salvador; M. A. Alonso \& M. Rodríguez 410 (USM).

Suele establecerse en quebradas y riberas.

Distribución.- hasta el momento restringida a los Andes de Bolivia y Perú. En Perú citada para los departamentos de Ancash, Apurímac, Cusco, Junín, La Libertad, Pasco.

\section{IRIDACEAE}

\section{Orthrosanthus occissapungus (Ruiz ex Klatt) Diels}

PERÚ, Huánuco: laguna Lauricocha, 3845 m, 28.IX.2006, F. Salvador; J. Monerris \& R. Soto $1009(\mathrm{ABH})$.

Habita llanuras y prados asociados a lagunas.

Distribución.- registrada en los Andes de Argentina, Bolivia y Perú. En el Perú citada para los departamentos de Ancash, Apurímac, Ayacucho, Cajamarca, La Libertad, Piura y Puno.

\section{JUNCACEAE}

\section{Luzula vulcanica Liebm.}

PERÚ, Huánuco: a $50 \mathrm{~m}$ de laguna Caballococha, orilla norte, 4580 m, 14.V.2004, F. Salvador; S. Rios \& E. Arias 996 (USM).

Se desarrolla en praderas turbosas y turberas dominadas por Distichia muscoides.

Distribución.- Mesoamericana (montaña)-Andina. En los Andes se registra en los territorios de Bolivia, Ecuador y Perú. En Perú se cita para los departamentos de Ancash, Lima y Puno.

\section{LOASACEAE}

\section{Calophora cirsiifolia C. Presl}

PERÚ, Huánuco: laguna Lauricocha, lado suroeste, $3845 \mathrm{~m}$, 7.VIII.2002, F. Salvador; M. A. Alonso \& M. Rodríguez 416 (USM).

Suele habitar en laderas pedregosas, suelos nitrificados y cultivos abandonados.

Distribución.- endemismo de la provincia de la Puna Peruana. Exclusiva de los Andes peruanos. Citada para los departamentos de Ancash, Apurímac, Cusco, Junín, Lima y Puno.

Estatus de conservación.- LC.

\section{Malvaceae}

\section{Nototriche longirostris (Wedd.) A.W. Hill}

PERÚ, Huánuco: campamento Raura, laguna Santa Ana, 4681 m, 7.V.2004, F. Salvador; S. Ríos \& E. Arias 728 (USM).

Forma parte de la revegetación realizada en márgenes lacustres situados sobre $4500 \mathrm{~m}$.

Distribución.- restringida a los Andes de Bolivia y Perú. En Perú citada para los departamentos de Ancash, Arequipa, Ayacucho, Cusco, Huancavelica, Junín, Pasco y Puno.

\section{OrCHidACEAE}

\section{Myrosmodes paludosa (Rchb. f.) C. Vargas}

PERÚ, Huánuco: a $100 \mathrm{~m}$ de puesto de control Santa Rosa (campamento Raura), 4650-4700 m, 13.V.2004, F. Salvador; S. Rios \& E. Arias 950 (USM).

Frecuentemente colectada en turberas dominadas por Distichia muscoides.

Distribución.- registrada para los territorios de Bolivia, Colombia, Perú y Venezuela. Escasamente recolectada en el Perú, citada para los departamentos de Ancash y La Libertad. 


\section{Orobanchaceae}

48. Bartsia patens Benth.

PERÚ, Huánuco: laguna Patarcocha, 4100 m, 11.VIII.2003, F. Salvador; M. A. Alonso \& J. Monerris 599 (USM); laguna Tinquicocha, orilla noroeste, $4350 \mathrm{~m}$.

Habita en turberas de Plantago tubulosa, también se ha observado en prados asociados a riachuelos.

Distribución.- citada para los Andes de Bolivia, Ecuador y Perú. En el Perú se registra en los departamentos de Ancash, Ayacucho, Cajamarca, Huancavelica, La Libertad, Lima y Pasco.

\section{Bartsia pedicularoides Benth.}

PERÚ, Huánuco: laguna Caballococha, orilla suroeste, 4575 m, 8.V.2004, F. Salvador; S. Ríos \& E. Arias 746 (USM); 9.V.2004, F. Salvador; S. Ríos \& E. Arias 786 (USM), laguna Pucacocha, nacimiento de río Antacallanca, $4350 \mathrm{~m}$, 11.V.2004, F. Salvador; S. Ríos \& E. Arias 845 (USM).

Se desarrolla en turberas de Distichia muscoides.

Distribución.- distribuida en los Andes de Bolivia, Colombia, Ecuador, Perú y Venezuela. En el Perú citada para los departamentos de Ancash, Arequipa, Cusco, Junín, Lima, Pasco y Puno.

\section{POACEAE}

\section{Agrostis breviculmis Hitchc.}

PERÚ, Huánuco: carretera a Jesús, a $3 \mathrm{~km}$ de comunidad campesina Lauricocha, 3950-4000 m, 10.V.2004, F. Salvador; S. Ríos \& E. Arias 816 (USM); ibidem, Salvador; S. Rios \& E. Arias 829 (USM); a $100 \mathrm{~m}$ de puesto de control Santa Rosa (campamento Raura), ladera arriba, 4700-4750 m, 13.V.2004, F. Salvador; S. Ríos \& E. Arias 921 (USM).

Habita laderas arenoso-pedregosas, prados y turberas en desecación.

Distribución.- registrada en los territorios de Argentina, Bolivia, Brasil, Chile, Colombia, Ecuador y Perú. En Perú citada para los departamentos de Amazonas, Ancash, Ayacucho, Cajamarca, Cusco, Huancavelica, Junín, La Libertad y Puno.

\section{Agrostis glomerata (J. Presl) Kunth}

PERÚ, Huánuco: río Lauricocha, 3326 m, 8.VIII.2003, F. Salvador; M. A. Alonso \& J. Monerris $542 a$ (USM).

Se encuentra en riberas y en laderas rocosas con suelo húmedo.

Distribución.- hasta el momento circunscrita a los territorios de Ecuador y Perú. En Perú citada para los departamentos de Ancash, Cajamarca, Cusco, Junín, Pasco y Puno.

52. Agrostis haenkeana Hitchc.

PERÚ, Huánuco: laguna Caballococha, orilla suroeste. 4575 m. 8.V.2004. F. Salvador; S. Ríos \& E. Arias 758b; oconal laguna Huascacocha, 4250 m, 11.V.2004, F. Salvador; S. Ríos \& E. Arias 852a (ABH, USM).

Escasamente colectada suele desarrollarse en turberas de Distichia muscoides.
Distribución.- distribuida en los territorios de Bolivia, Colombia, Ecuador y Perú. En Perú citada para los departamentos de Ancash, Cajamarca, Cusco, Huancavelica, La Libertad, Junín y Puno.

\section{Bromus catharticus Vahl}

PERÚ, Huánuco: río Lauricocha, 3326 m, 8.VIII.2003, F. Salvador; M. A. Alonso \& J. Monerris 541c (USM); laguna Pucacocha, 4350 m, 11.V.2004, F. Salvador; S. Rios \& E. Arias $846 b(\mathrm{ABH})$.

Crece en laderas rocosas y zonas de riberas.

Distribución.- cosmopolita. Ampliamente distribuida en el Perú.

\section{Calamagrostis jamesoni Steud.}

PERÚ, Huánuco: laguna Patarcocha, orilla oeste, $4100 \mathrm{~m}$, 12.V.2004, F. Salvador; S. Ríos \& E. Arias 883 (USM); a 100 $\mathrm{m}$ de puesto de control Santa Rosa (campamento Raura), ladera arriba, 4700-4750 m, 13.V.2004, F. Salvador; S. Ríos \& E. Arias 928a (USM); a $70 \mathrm{~m}$ de puesto de control Santa Rosa (campamento Raura), vertiente Atlántica, 4650-4700 m, 13.V.2004, F. Salvador; S. Ríos \& E. Arias 938 (ABH, USM).

Se ha observado mayormente creciendo en márgenes de riachuelos y lagunas. En otros casos también crece en turberas de Distichia muscoides.

Distribución.- distribuida en los Andes de Bolivia, Colombia, Ecuador y Perú. En Perú citada para los departamentos de Ancash, Huancavelica, Lima y Puno.

\section{Calamagrostis vicunarum (Wedd.) Pilg.}

PERÚ, Huánuco: laguna Caballococha, orilla suroeste. 4575 m. 8.V.2004, F. Salvador; S. Rios \& E. Arias 758c (ABH).

Escasamente colectada, suele desarrollarse en turberas de Distichia muscoides.

Distribución.- registrada en los territorios andinos de Argentina, Bolivia, Chile, Ecuador y Perú. En el Perú citada para los departamentos de Ancash, Ayacucho, Cusco, Huancavelica, Junín, Lima, La Libertad, Pasco, Puno y Tacna.

\section{Cortaderia planifolia Swallen}

PERÚ, Huánuco: laguna Pucacocha, nacimiento de río Antacallanca, 4350 m, 11.V.2004, F. Salvador; S. Rios \& E. Arias 836 (USM).

Escasamente colectada, crece en zonas de desagüe de lagunas con fuerte estiaje.

Distribución.- hasta el momento restringida a los Andes de Colombia y Perú. Escasamente colectada en el Perú. Citada para Ancash, Huancavelica, Junín y Lima.

\section{Dactylis glomerata L}

PERÚ, Huánuco: laguna Lauricocha, orilla suroeste, $3845 \mathrm{~m}$, 7.VIII.2002, F. Salvador; M. A. Alonso \& M. Rodríguez 400 (USM).

Especie nitrófila que se desarrolla en antiguos cultivos abandonados.

Distribución.- introducida. Esta especie es considerada como maleza o planta invasora (cf. Stubbendieck et al., 1994; 
Uva et al., 1997). En Perú citada para Cajamarca, Cusco, Huancavelica, Huancavelica y La Libertad.

\section{Festuca dichoclada Pilg.}

PERÚ, Huánuco: subida a laguna Tactapata, $4000 \mathrm{~m}$, 12.VIII.2003, F. Salvador; M. A. Alonso \& J. Monerris 638 (ABH, USM).

Escasamente colectada, suele crecer en zonas de riberas.

Distribución.- hasta la actualidad exclusiva de los Andes de Ecuador y Perú. En Perú citada para los departamentos de Ancash, Cajamarca, Cusco, Huancavelica, Junín, La Libertad y Lima.

\section{Festuca hieronymi Hack.}

PERÚ, Huánuco: laguna Patarcocha, orilla oeste, $4100 \mathrm{~m}$, 12.V.2004, F. Salvador; S. Ríos \& E. Arias 885a (USM).

Escasamente colectado, se desarrolla en turberas de Plantago tubulosa.

Distribución.- citada para los Andes de Argentina, Bolivia y Perú. En el Perú registrada hasta el momento en los departamentos de Apurímac, Cusco y Huancavelica.

\section{Poa glaberrima Tovar}

PERÚ, Huánuco: campamento Raura, laguna Niñococha bajo, 4795 m, 7.V.2004, F. Salvador; S. Rios \& E. Arias 734 (USM).

Escasamente colectada, suele desarrollarse en márgenes de lagunas con suelos arenosos.

Distribución.- hasta la actualidad exclusiva de los Andes de Bolivia y Perú. En Perú citada para los departamentos de Ancash, Huancavelica y Junín.

\section{Stipa brachyphylla Hitchc.}

PERÚ, Huánuco: laguna Patarcocha, orilla oeste, $4100 \mathrm{~m}$, 12.V.2004, F. Salvador; S. Ríos \& E. Arias 8856 (USM).

Raramente observada, se desarrolla en turberas dominadas por Plantago tubulosa.

Distribución.- registrada en los territorios andinos de Argentina, Bolivia, Colombia, Ecuador y Perú. En Perú citada para los departamentos de Ancash, Ayacucho, Cajamarca, Cusco, Huancavelica, Junín, Lima, Pasco y Puno.

\section{Plantaginaceae}

\section{Plantago australis Lam.}

PERÚ, Huánuco: laguna Lauricocha, lado norte, 3845 m, 11.VIII.2002, F. Salvador; M. A. Alonso \& M. Rodríguez 534 (USM); Jesús, río Lauricocha, 3326 m, 8.VIII.2003, F. Salvador; M. A. Alonso \& J. Monerris 556 (USM), subida a laguna Tactapata, $4050 \mathrm{~m}$, 12.VIII.2003, F. Salvador; M. A. Alonso \& J. Monerris 638 (USM).

Encontrada en turberas y bajo matorrales en suelos ligeramente húmedos.

Distribución.- Neotropical-Austroamericana. En Perú citada para los departamentos de Amazonas y Cajamarca, con esta colección se amplia la distribución hacia la zona central.

\section{Plantago orbygniana Steinh. ex Decne.}

PERÚ, Huánuco: carretera a Jesús, a 1,5 km de comunidad campesina Lauricocha, 3950-4000 m, 10.V.2004, F. Salvador; S. Ríos \& E. Arias 812 (USM).

Suele desarrollarse en laderas arenoso-pedregosas en suelos con ligera hidromorfía.

Distribución.- distribuida en los territorios de Argentina, Bolivia, Colombia, Ecuador y Perú. En Perú citada para los departamentos de Arequipa, Junín y Puno.

\section{Polygonaceae}

\section{Rumex acetosella $\mathrm{L}$.}

PERÚ, Huánuco: río Lauricocha, 3326 m, 08/08/2003, F. Salvador; M. A. Alonso \& J. Monerris 562 (USM); río Antacallanca, entre laguna Tinquicocha y Chuspi, $4250 \mathrm{~m}$, 12.V.2004, F. Salvador; S. Ríos \& E. Arias 899 (USM).

Crece en prados asociados a riberas, también se ha observado bajo matorrales.

Distribución.- cosmopolita. En Perú citada para los departamentos de Ancash, Cajamarca, Cusco, Junín, La Libertad y Puno.

\section{Rumex peruanus Rech. $\mathrm{f}$.}

PERÚ, Huánuco: Añaspampa, 3915 m, 12.V.2004, F. Salvador; S. Rios \& E. Arias 915 (USM). Salvador; M. A. Alonso \& M. Rodríguez 380 (USM).

Escasamente colectada, crece en zonas pantanosas de valles fluvio-lacustres y en riachuelos de poca corriente.

Distribución.- restringida a los Andes de Colombia, Ecuador y Perú. En Perú citada para los departamentos de Amazonas, Ancash, Cajamarca, Cusco y Junín.

\section{Ranunculaceae}

\section{Ranunculus flagelliformis $\mathrm{Sm}$.}

PERÚ, Huánuco: riachuelo cerca de laguna Taulicocha, 3910 m, 11.VIII.2002, F. Salvador; M. A. Alonso \& M. Rodríguez $457 a$ (USM); oconal de Ańaspampa, a $5 \mathrm{~km}$ de Antacallanca, 4000 m, 14.VIII.2003, F. Salvador; M. A. Alonso \& J. Monerris 705a (USM); a $1 \mathrm{~km}$ de Antacallanca, 4000 m, 12.V.2004, F. Salvador; S. Ríos \& E. Arias 901 (USM).

Ocupa orillas superficiales de lagunas, riachuelos y charcas temporales.

Distribución.- Neotropical-Austroamericana. En Perú se cita para los departamentos de Amazonas, Ancash, Ayacucho, Cajamarca, Cusco, Junín y La Libertad.

\section{Ranunculus limoselloides Turcz.}

PERÚ, Huánuco: laguna Taulicocha, orilla este, $3910 \mathrm{~m}$, 8.VIII.2002, F. Salvador; M. A. Alonso \& M. Rodríguez 502 (USM).

Propio de orillas superficiales de lagunas y riachuelos, en general en aguas con poca corriente.

Distribución.- distribuida en los Andes de Bolivia, Colombia, Ecuador y Perú. En Perú se cita para los departamentos de Ancash, Cajamarca, Cusco, La Libertad y Tacna. 
68. Ranunculus trichophyllus Chaix ex Vill.

PERÚ, Huánuco: laguna Caballococha, 4550 m, 13.VIII.2003, F. Salvador; M. A. Alonso \& J. Monerris 693 (USM); riachuelo oconal de Añaspampa, a $5 \mathrm{~km}$ de Antacallanca, $4000 \mathrm{~m}$, 14.VIII.2003, F. Salvador; M. A. Alonso \& J. Monerris 706 (USM); laguna Niño Perdido, 4734 m, 14.V.2004, F. Salvador; S. Ríos \& E. Arias 1002 (USM).

Se desarrolla en aguas superficiales de lagunas.

Distribución.- Holártica-Andina. Distribuida en los Andes de Argentina, Bolivia, Chile, Perú. En Perú se cita para los departamentos de Arequipa, Loreto y Puno.

\section{RosaceAe}

69. Alchemilla andina (L.M. Perry) J.F. Macbr.

PERÚ, Huánuco: Jesús, río Lauricocha, 3326 m, 9.VIII.2003, F. Salvador; M. A. Alonso \& J. Monerris 575 (USM).

Suele habitar prados asociados a ríos, también se ha observado creciendo bajo matorrales.

Distribución.- hasta la actualidad exclusiva de los territorios de Ecuador y Perú. Escasamente colectada. Se cita por el momento en el departamento de La Libertad, con esa cita se extiende su distribución hacia los Andes centrales peruanos.

\section{Alchemilla diplophylla Diels}

PERÚ, Huánuco: laguna Tinquicocha, orilla noroeste, 4350 m, 9.V.2004, F. Salvador; S. Rios \& E. Arias 780 (USM).

Ampliamente observada, suelo ocupar suelos encharcados y turberas.

Distribución.- localizada en los Andes de Argentina, Bolivia, Chile y Perú. En el Perú citada para los departamentos de Ancash, Cusco, Junín y Lima.

\section{RUBiACEAe}

71. Galium pumilio Standl.

PERÚ, Huánuco: borde de laguna Lauricocha, $3845 \mathrm{~m}$, 5.VIII.2002, F. Salvador; M. A. Alonso \& M. Rodríguez 368 (MO); laguna Patarcocha, 4100 m, 11.VIII.2003, F. Salvador; M. A. Alonso \& J. Monerris 586 (USM).

Suele crecer en prados asociados a riachuelos, algunas ocasiones se ha observado en turberas de Plantago tubulosa.

Distribución.- hasta el momento restringida a los Andes de Ecuador y Perú. Escasamente colectada en el Perú, citada para el departamento de Pasco.

\section{RupPIACEAE}

\section{Ruppia filifolia (Phil.) Skottsb.}

PERÚ, Huánuco: laguna Niño Perdido, ladera este, 4734 m, 14.V.2004, F. Salvador; S. Ríos \& E. Arias 1003 (USM); laguna Puyhuancocha, ladera oeste, 17.IX.2005, F. Salvador; M. Epiquién \& R. García 1004 (ABH, USM).

Se ha registrado en aguas superficiales de lagunas y acequias situadas sobre $4500 \mathrm{~m}$.

Distribución.- distribuida en los Andes de Argentina, Bolivia, Chile y Perú. En el Perú citada para los departamentos de Cajamarca, Cusco, Junín, Puno y San Martín.

\section{SAXIFRAGACEAE}

\section{Saxifraga magellanica Poir.}

PERÚ, Huánuco: laguna Lauricocha, lado suroeste, $3845 \mathrm{~m}$, 8.VIII.2002, F. Salvador; M. A. Alonso \& M. Rodríguez 423 (USM); ladera rocosa laguna Tactapata segunda, $4310 \mathrm{~m}, 12$.VIII.2003, $F$. Salvador; M. A. Alonso \& J. Monerris 647 (USM).

Especie casmófita crece sobre rocas verticales.

Distribución.- registrada para los Andes de Argentina, Bolivia, Chile, Ecuador y Perú. En el Perú citada para Ancash, Apurímac, Cajamarca, Cusco, Junín, Lima, La Libertad y Puno.

\section{SCROpHULARIACEAE}

\section{Ourisia muscosa Benth.}

PERÚ, Huánuco: laguna Tinquicocha, 4000 m, 13.VIII.2003, F. Salvador; M. A. Alonso \& J. Monerris 667 (USM); ibidem, F. Salvador; M. A. Alonso \& J. Monerris $668^{\circ}$ (USM); laguna Huascacocha, 4250 m, 11.V.2004, F. Salvador; S. Ríos \& E. Arias 849 (USM); a $100 \mathrm{~m}$ de puesto de control Santa Rosa (campamento Raura), ladera arriba, 4700-4750 m, 13.V.2004, F. Salvador; S. Ríos \& E. Arias 925 (USM); a $50 \mathrm{~m}$ de laguna Caballococha, orilla norte, 4580 m, 13.V.2004, F. Salvador; S. Ríos \& E. Arias 972 (USM).

Especie propia de comunidades turfófilas dominadas por Distichia muscoides.

Distribución.- localizada en los territorios de Argentina, Bolivia, Ecuador y Perú. En Perú registrada en los departamentos de Ancash, Arequipa y Puno. Con esta colección se amplia su presencia en los Andes centrales.

\section{VALERIANACEAE}

75. Valeriana condamoana Graebn.

PERÚ, Huánuco: a $100 \mathrm{~m}$ de puesto de control Santa Rosa (campamento Raura), ladera abajo, 4650-4700 m, 13.V.2004, F. Salvador; S. Ríos \& E. Arias 951 (USM).

Crece en turberas dominadas por Distichia muscoides y Plantago rigida, localizadas por encima de $4600 \mathrm{~m}$.

Distribución.- exclusiva de los Andes de Bolivia y Perú. En Perú citada para los departamentos de Ancash, Huancavelica y Junín.

\section{Agradecimientos}

Por los aportes y revisiones al manuscrito agradecemos a Barbara Ruthsatz (Universidad de Trier, Alemania), Blanca León (Universidad de Texas, USA) y a los editores y revisores anónimos. El Jardín Botánico del Missouri otorgó una beca Bascom a la primera autora, la cual permitió la visita a los herbarios $\mathrm{F}$, $\mathrm{MO}, \mathrm{NY}$ y MICH. Por la revisión y determinación del material conflictivo, agradecemos a Hamilton Beltrán (USM), Blanca León (USM, TEX), T. Koyama (MBK), Ulf Molau (GB), Anton Reznicek (MICH), Al Shebazh (MO), Oscar Tovar (USM) y a G. Wheeler (MIN). El trabajo de campo ha sido posible gracias a la colaboración del Asunción Cano (USM), Maritza Rodríguez, Mirbel Epiquién, Jorge Monerris, Enrique Arias y Renzo García. Este trabajo ha sido financiado por el Fondo de Humedales para el Futuro de la Convención Ramsar y la Universidad de Alicante. 


\section{Literatura citada}

Al-Shehbaz I. A. 1990. A revision of Weberbauera (Brassicaceae). Journal of the Arnold Arboretum 71(2): 221-250.

Al-Shehbaz I. A. 2004. A synopsis of the South American Weberbauera (Brassicaceae). Novon 14(3): 258-268.

Brako L. \& J. L. Zarucchi. 1993. Catalogue of the Flowering Plants and Gymnosperms of Peru. Monographs Sys. Bot. Missouri Bot. Gard. 45, 1-1286.

Dhooge S. \& P. Goetghebeur. 2002. A new Andean species and a new combination in Oreobolopsis (Cyperaceae). Novon 12 (3): 338-342.

Dhooge S. \& P. Goetghebeur. 2004. Phylloscirpus (Cyperaceae) revisited. Novon 14(3): 278-284.

Dhooge S., P. Goetghebeur \& A.M. Muasya. 2003. Zameioiscirpus, a new genus of Cyperaceae from South America. Plant Systematics and Evolution 243: 73-84.

Koyama T. \& E. R. Guaglianone. 1987. Oreobolopsis, a new genus of Cyperaceae (Scirpeae) from Bolivia, South America. Darwiniana 28: 79-85.

León B., J. Roque, C. Ulloa, N. Pitman, P.M. Jørgensen \& A. Cano (eds). 2006. Libro rojo de especies endémicas del Perú. Rev. peru. biol. Número especial 13(2).

Macbride J. F. 1936-1967. Flora of Peru. Field Mus. Nat. Hist. Bot. Ser.13.

Missouri Botanical Garden. 2007. W3Tropicos. Missouri Botanical Garden's VAST (VAScular Tropicos). <http:// mobot.mobot.org/W3T/Search/vast.html. >. Consulta: $[25 / 05 / 2007)$.

Reznicek A. A. 1993. Revision of Carex section Ovales (Cyperaceae) in Mexico. Contributions from the University of Michigan Herbarium 19: 97-136.

Rivas-Martínez S., D. Sanchez-Mata \& M. Costa. 1999. North American boreal and western temperate forest vegetation. Itinera Geobotanica 12: 5-316.
Salvador F.M. \& A. Cano. 2002. Lagunas y oconales: los humedales del Trópico Andino. Cuadernos de Biodiversidad 11: 4-9. Centro Iberoamericano de la Biodiversidad (CIBIO), Universidad de Alicante.

Salvador F.M., M.A. Alonso \& S. Ríos. 2006. Adiciones a la flora andina peruana del departamento de Huánuco I. Candollea 61 (2): 279--291.

Salvador F.M. 2008. Paisaje vegetal de los humedales de una cuenca altoandina: subcuenca del río Lauricocha, Huanuco, Andes centrales, Perú. Tesis Doctoral inédita. Universidad de Alicante. España. 522 pp.

Seberg O. 1994. Oreobolus. Flora Mesoamericana 6: 422-423. Universidad Nacional Autónoma de México, México, D. F.

Stubbendieck J., G.Y. Friisoe, \& M.R. Bolick. 1994. Weeds of Nebraska and the Great Plains. Nebraska Department of Agriculture, Bureau of Plant Industry. Lincoln, Nebraska. $589 \mathrm{pp}$.

UICN. Unión Mundial para la Conservación de la Naturaleza. 2001. Las Categorías de la Lista Roja de la UICN. < http:// www. iucn.org/themes/ssc/redlists/rlcategories 2000.html>. [Consulta: 02/07/2007].

Uva R.H., J.C. Neal, \& J.M. DiTomaso. 1997. Weeds of the Northeast. Cornell University Press. Ithaca, New York. $397 \mathrm{pp}$.

Wheeler G.A. 1989. A new species of Carex sect. Abditispicae (Cyperaceae) from northern Argentina and the status of Vesicarex collumanthus in South America. Systematic Botany 14(1): 37-42.

Wheeler G.A. 2002. A new species of Carex Section Abditispicae (Cyperaceae) from South America and additional notes on the Section. Darwiniana 40(1-4): 191-198. 